\title{
ANÁLISIS DE LA COLABORACIÓN CIENTÍFICA EN LA UNIVERSIDAD POLITÉCNICA DE VALENCIA
}

\author{
ANALYSIS OF SCIENTIFIC COLLABORATION AT THE POLYTECHNIC UNIVERSITY OF VALENCIA \\ Arnau Fombuena \\ Department of Spatial Information Engineering, Namseoul University, 91 Daehak-ro, Seonghwan-eup, Seobuk-gu, Chungcheongnam- \\ do, 331-707 Cheonan, South Korea. fombuena@nsu.ac.kr
}

\begin{abstract}
:
The work presented in this paper is focused on employing maps for the visualization of the collaboration network of the Polytechnic University of Valencia (Spain). In this paper, a collaboration is understood as the scientific publications where at least one author is affiliated in the aforementioned university. Furthermore, a visual analysis using the maps created is performed with the goal of observing the proximity of the institutions collaborating with said university, the intensity of said collaborations, and supporting future decision-making processes for new research projects.
\end{abstract}

Key words: Geomatics, GIS, Higher Education

\section{Resumen:}

El trabajo presentado en este artículo se centra en la visualización de la red de instituciones con las que colabora la Universidad Politécnica de Valencia a través de mapas. Entendiendo como colaboraciones las publicaciones científicas en las que al menos un autor pertenece a dicha institución. Además, se expone un análisis visual mediante los mapas creados con el objetivo final de observar la proximidad de las instituciones colaboradoras con la universidad y la intensidad de las colaboraciones, que puedan servir de apoyo a la toma de decisiones para futuros proyectos de investigación.

Palabras clave: Geomática, SIG, Educación Superior

\section{Introducción}

La educación es un factor esencial para el desarrollo social y económico de la sociedad (Rumbley et al. 2014). La educación superior en particular debe enfrentar nuevos desafíos y funciones que tradicionalmente no han sido interpretadas como propias. En este sentido, la Unión Europea (UE) se ha apoyado en la expansión de la implicación de las Instituciones de Educación Superior (IES) en la sociedad como pilar fundamental para desarrollar la sociedad del conocimiento (European Commission 2011). La estrategia europea Horizon2020 (European Commission 2014) es una muestra significativa del aumento de la importancia de las IES y de la necesidad de comprender, gestionar, planificar y apoyar la toma de decisiones eficaz en el sector de la educación superior. Sin embargo, el actual ritmo acelerado de creación y almacenamiento de datos conlleva que la extracción de valor añadido sea un proceso complejo (Turi et al. 2013). Asimismo, los datos geoespaciales también han seguido esta tendencia y están ganando importancia convirtiéndose en big data por derecho propio, dando paso a la big geo-data (Sui 2014). Debido a la relevancia de los datos geoespaciales Naciones Unidas creó en 2011 el United Nations Committee of Experts on Global Geospatial Information Management (2014) con el objetivo de gestionar los múltiples desafíos que presenta la situación actual a nivel global en lo relativo a estándares, instrumentos legales, datos de infraestructuras espaciales, etc.
Autores como Goodchild y Janelle (2010) destacan la importancia de los patrones geoespaciales para mejorar los planes estratégicos y ven los Sistemas de Información Geográfica (SIG) como la pieza esencial para aunar la perspectiva interdisciplinar. Otros autores como Jessop (2008) y Boonstra (2009) consideran indispensable la información geoespacial en el campo de las humanidades aunque reconocen la lenta adaptación de los SIG a las necesidades de la investigación en dicho campo. La propuesta de Esri (Sui 2014) para crear una Universidad Espacial centrada en explotar al máximo el potencial de las tecnologías geoespaciales representa una oportunidad para hacer de los SIG un elemento indispensable en las IES. Concretamente, esta propuesta se basa en cuatro aspectos principales:

1. Expandir el uso del Spatial Thinking (SP) como piedra angular de los planes de estudios. Varios autores (Bearman et al. 2015; Ellul 2015; Ferrandino 2015; Xie and Reider 2014) han realizado diversos estudios en los que concluyen que aprender SP contribuye a aumentar la probabilidad de encontrar empleo una vez acabados los estudios universitarios. Sobre todo para los estudiantes de carreras no técnicas.

2. Impulsar una línea de trabajo con formación geoespacial que sea capaz de aprovechar las oportunidades laborales asociadas con la tecnología geoespacial (Gewin 2004; US Department of Labor 2010). 
3. Posibilitar e impulsar la investigación académica interdisciplinar tomando como referencia la perspectiva geoespacial.

4. Desarrollar smart campuses cuya gestión de recursos y espacios se vea reforzada con SIG.

Resumiendo, lo que se propone es incluir los SIG en los planes de estudios universitarios - más allá de aquellos específicamente centrados en la geomática-, promover la investigación con datos geoespaciales, mejorar la gestión de las universidades a través de los SIG y apoyar la toma de decisiones. Todo ello, integrando los SIG en la universidad de una forma similar a la estadística que, hoy en día, es indiscutible en muchísimos ámbitos, sean éstos científicos o no. Particularmente, la complejidad de los múltiples aspectos de la educación superior lleva a la necesidad de aportar mejoras a las limitaciones de la estadística (Pérez-Gómez 2012). Además, muchos de los estudios realizados en diversos campos presentan la información de un modo que frecuentemente resulta tan complicado como los propios estudios. Esta situación implica que dichos estudios no se exploten al máximo. En este sentido, la inclusión de los SIG es una evolución natural con el potencial de enriquecer los estudios típicamente estadísticos.

El contexto de la educación superior, debido a su complejidad y a sus enormes dimensiones, resulta especialmente exigente en la recopilación de datos, la gestión y el análisis de sus múltiples aspectos. Es por ello que ha habido algunas iniciativas para crear sistemas de apoyo al proceso de toma de decisiones en la educación superior empleando los SIG. Los tres sistemas brevemente descritos a continuación destacan significativamente:

1. Los responsables del sistema universitario del estado de Oregón (2004) y la Oregon State University, en los Estados Unidos de América, decidieron realizar un programa piloto llamado Oregon State Board of Education Decision-Support Pilot Project (OSBEDSPP). El objetivo era analizar datos demográficos y estadísticos con SIG para apoyar la toma de decisiones. Específicamente, para la financiación de las universidades y el desarrollo de la fuerza laboral.

2. Wang et al. (2009) desarrollaron un sistema en la Peking University con el objetivo principal de facilitar la transparencia del proceso de toma de decisiones al público en general. Además, a través de este sistema los investigadores y los responsables de toma de decisiones reciben de forma sencilla información de varias fuentes, que resulta útil para el desempeño de sus funciones.

3. Singleton et al. (2012) modelaron la participación en la Educación Superior con un modelado espacial interactivo conectado a la clasificación demográfica. El objetivo principal era poder observar la aplicación hipotética de diversas políticas. Particularmente, simulando cómo se vería afectada la participación y considerando cada institución y cada tipo de barrio.

Si bien estas iniciativas resultan interesantes es importante tener en cuenta que tienen un carácter altamente experimental y la expansión, o incluso el conocimiento, de este tipo de sistemas todavía no se han desarrollado. Ésta última afirmación resulta evidente al observar la existencia de trabajos con el objetivo de apoyar la toma de decisiones que no utilizan los SIG. Por ejemplo, analizando tendencias educacionales por zonas y en una escala multitemporal (Smith and Smith 2014)

En particular, en este artículo se muestra la red de colaboradores de la Universidad Politécnica de Valencia (UPV) en el año 2015 a partir de datos obtenidos de la base de datos científica Scopus. El objetivo es doble: 1) visualizar a través de una red los colaboradores de la UPV para, después, realizar un análisis visual de dicha red. Este artículo sigue una estructura clásica pero efectiva para transmitir conocimiento científico y presenta la situación actual de los SIG en la educación superior. 2) Detallar el método utilizado para satisfacer el objetivo propuesto.

A continuación, se presenta el método seguido y los resultados para, finalmente, exponer las conclusiones obtenidas.

\section{Método}

Para conseguir la realización del objetivo se requieren datos de artículos científicos. A través de la colaboración entre autores en publicaciones científicas se deriva la colaboración científica entre las entidades a las que dichos autores pertenecen. Para obtener la red de instituciones que colaboran con la UPV se han tomado aquellas publicaciones en las que al menos un autor pertenece a la UPV.

Las publicaciones científicas están accesibles a través de las webs de las revistas que los publican así como en bases de datos científicas. Visitar todas y cada una de las revistas científicas existentes resultaría complicado y tedioso. En su lugar, se ha accedido a las publicaciones a través de las bases de datos científicas.

Existen muchas bases de datos científicas. Pero hay dos consideradas de mayor calidad, la Web of Science (WoS) y Scopus, porque indexan las revistas con mejor reputación que, además, cumplen con el sistema de revisión por pares para asegurar la calidad de los artículos publicados.

Entre la base de datos de la WoS y la de Scopus se ha elegido la segunda. El motivo de esta elección reside en que la base de datos de la WoS es sustancialmente más restrictiva debido a que únicamente incluye aquellas revistas que han sido previamente indexadas por Clarivate Analytics (antes Thomson Reuters) y que, por tanto, han recibido un factor de impacto y han sido clasificadas en una de sus múltiples categorías. La base de datos de Scopus es más completa, porque además de las revistas incluidas en la WoS también incluye otras revistas que cumplen con una serie de criterios establecidos por Elsevier (n.d.), propietaria de Scopus, para garantizar la calidad y relevancia de dichas revistas. Es por ello que Scopus ofrece una visión más amplia de la ciencia que la WoS. Cabe destacar, que hasta 1996 aproximadamente la WoS ofrece una base de datos más completa pero a partir de esa fecha Scopus ofrece mejores resultados.

Una vez obtenidos los datos necesarios de Scopus, se ha creado una matriz numpy en la que se han 
almacenado el número de colaboraciones entre cada par de instituciones. Dichas colaboraciones se han calculado por pares de forma que si en un artículo científico participan tres autores $a, b, c$ pertenecientes a tres instituciones $x, y, z$, las colaboraciones resultantes serán las de la institución $x$ con $y$, la institución $x$ con $z y$ la institución $y$ con $z$. De esta forma, se obtendrá una red con forma poligonal para cada artículo en el caso de que participen tres o más instituciones. En el caso de que únicamente participen dos instituciones se obtendrá una línea de colaboración uniendo ambas instituciones.

Para visualizar las colaboraciones, se ha empleado ArcGIS. Con este software se ha creado una geodatabase en la que se ha almacenado una capa de puntos que contiene las instituciones a las que están afiliados los autores que firman las publicaciones científicas. Dichas instituciones se han geolocalizado previamente de forma automatizada empleando Python. Además, también se han limpiado los datos con el fin de evitar la aparición de instituciones duplicadas y corregir las coordenadas erróneas. Es decir, se han modificado las coordenadas de las instituciones que no hayan sido geolocalizadas correctamente. Además, se ha revisado y corregido el tipo de institución en los casos oportunos. La simbología de las instituciones en la capa de puntos se ha creado de modo que el color de cada punto sea distinto para cada tipo de institución. En la geodatabase también se ha creado una capa de líneas que une las instituciones que colaboran en la producción científica. La simbología de dichas líneas, concretamente el grosor, representa el número de colaboraciones entre cada par de instituciones.

\section{Resultados}

La matriz de colaboraciones tiene unas dimensiones de 1091 x 1091. Es decir, el total de instituciones que colaboran con la UPV es de 1091 que generan, en conjunto, un total de colaboraciones - a partir de las publicaciones científicas de la UPV indexadas en Scopus en el año 2015 - de 8304 (Fig. 1).

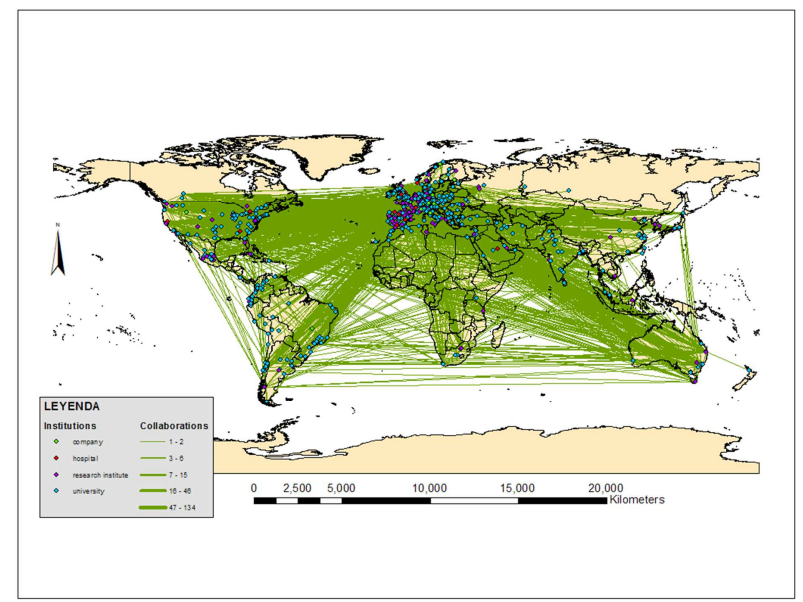

Figura 1: Red de colaboraciones generada a partir de publicaciones de la UPV en 2015.

El total de colaboraciones entre universidades y empresas generadas a partir de las publicaciones científicas de la UPV indexadas en Scopus en el año 2015 es de 357 (Fig. 2).

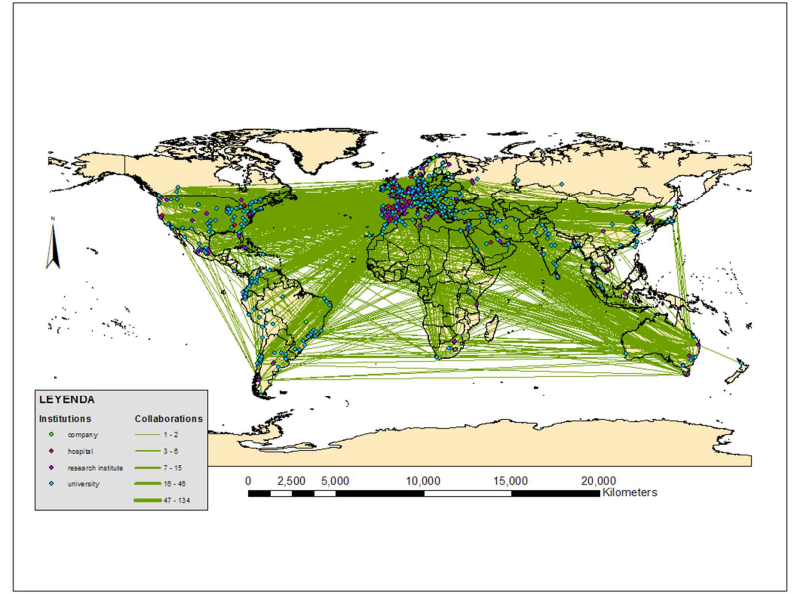

Figura 2: Red de colaboraciones entre universidades y empresas generada a partir de publicaciones de la UPV en 2015.

El total de colaboraciones entre universidades y hospitales generadas a partir de las publicaciones científicas de la UPV indexadas en Scopus en el año 2015 es de 266 (Fig. 3).

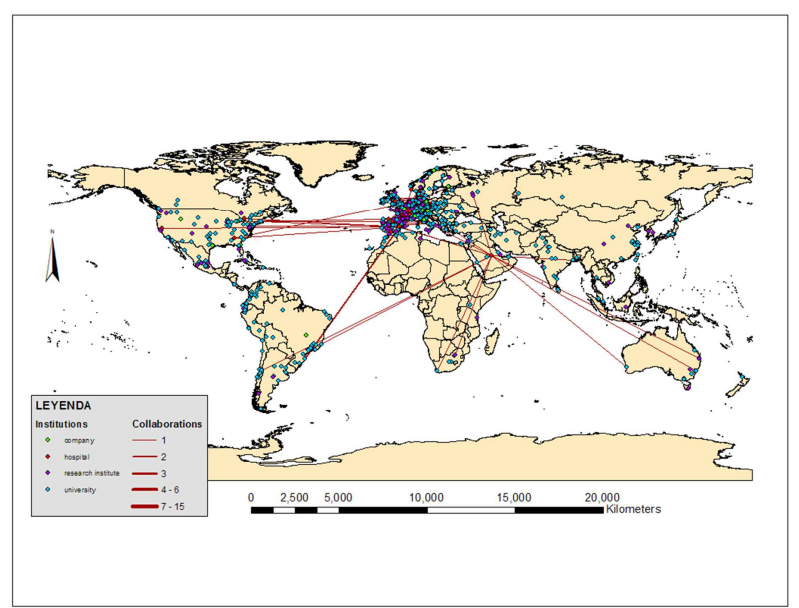

Figura 3: Red de colaboraciones entre universidades y hospitales generada a partir de publicaciones de la UPV en 2015.

El total de colaboraciones entre universidades y empresas generadas a partir de las publicaciones científicas de la UPV indexadas en Scopus en el año 2015 es de 2400 (Fig. 4).

El total de colaboraciones únicamente entre universidades generadas a partir de las publicaciones científicas de la UPV indexadas en Scopus en el año 2015 es de 2616 (Fig. 5).

\section{Conclusiones}

En este artículo se han mostrado las colaboraciones científicas de la Universidad Politécnica de Valencia en el año 2015 con otras instituciones. Para ello se han tomado datos de publicaciones en revistas científicas y se han extraído las colaboraciones de autores pertenecientes a distintas instituciones a partir de las publicaciones de la UPV. Además, se han explicitado dichas colaboraciones a través de mapas para, finalmente, realizar un análisis visual. 


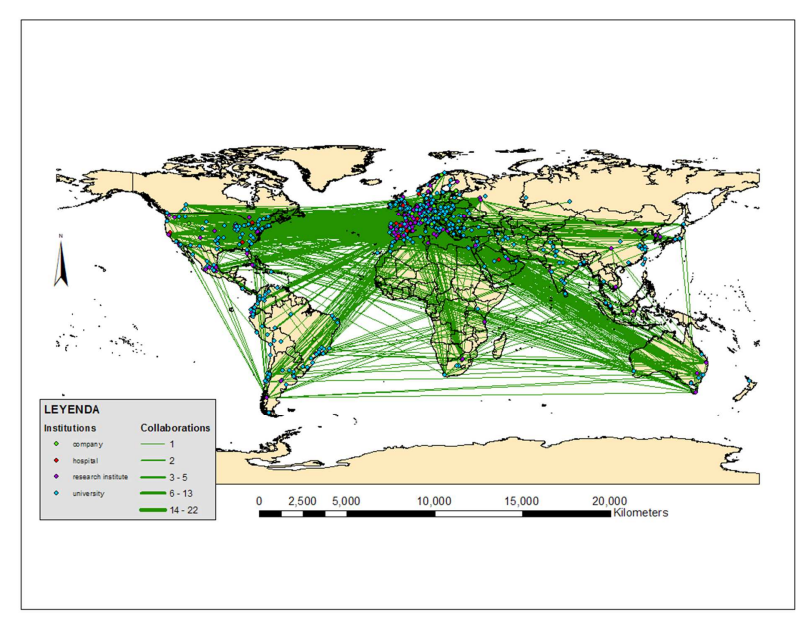

Figura 4: Red de colaboraciones entre universidades e institutos de investigación generada a partir de publicaciones de la UPV en 2015.

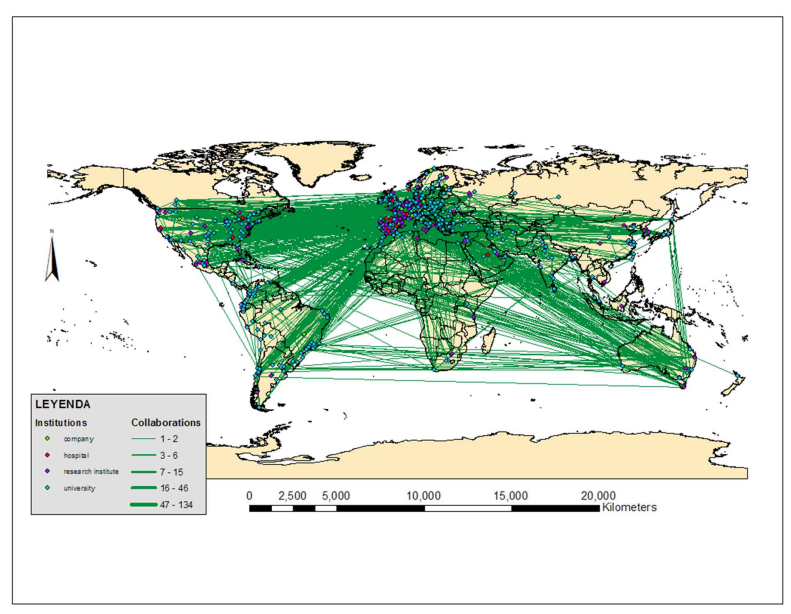

Figura 5: Red de colaboraciones únicamente entre universidades generada a partir de publicaciones de la UPV en 2015.

De los resultados del análisis visual se obtiene que la UPV colabora principalmente con otras universidades y con institutos de investigación. Aunque la UPV también colabora con empresas y hospitales, dichas colaboraciones son mucho más reducidas que en los dos casos citados anteriormente. Si bien, comparativamente, el número de colaboraciones con empresas es relativamente bajo, destaca su distribución geográfica en todos los continentes. El escaso número de colaboraciones con empresas y hospitales puede deberse a la naturaleza de las publicaciones científicas. Muchas empresas tienen interés en desarrollar productos y servicios pero no tanto en publicarlos en revistas científicas. Del mismo modo, los hospitales buscan soluciones que no necesariamente publican en revistas científicas. De hecho, las publicaciones científicas de los hospitales provienen, a menudo, de los institutos de investigación de los propios hospitales, por lo que se contabilizan en esa categoría. También cabe destacar que la colaboración de la UPV con hospitales asiáticos, así como con los de la gran mayoría de países africanos, es inexistente.

Respecto a la distribución geográfica, la UPV colabora con instituciones que están localizadas, mayoritariamente, en Europa. La UPV también colabora con muchas instituciones del continente americano, tanto en el norte como en el sur. En el sur las colaboraciones son principalmente con universidades, mientras que en el norte el tipo de instituciones con los que se relaciona la UPV es más variado, con una presencia mayor de institutos de investigación, hospitales y empresas. En el continente africano las colaboraciones están focalizadas en el norte y en el sur con prácticamente ninguna colaboración en el resto del continente. Muchos países de oriente medio están también representados en la red de colaboraciones de la UPV. Respecto a Asia, las colaboraciones se reparten entre la India y China con algunas colaboraciones en países del sudeste asiático, Japón y Corea del Sur. En la zona de Oceanía las principales instituciones del continente también son colaboradoras de la UPV. Éstas instituciones están localizadas, en su mayoría, en la costa este de Australia.

La intensidad de las colaboraciones con otras instituciones es muy moderada, con un volumen medio de colaboraciones por institución de 1,3 colaboraciones independientemente del tipo de institución. Sin embargo, las instituciones que más colaboran con la UPV son la Universidad de Valencia, con 134 colaboraciones y la Universitat Jaume I, con 46 colaboraciones. Ambas superan con creces el volumen medio comentado anteriormente. Esto ratifica que la primera ley de la geografía (Tobler 1970) se cumple en esta red de colaboraciones y que todo está relacionado entre sí, pero la relación es más intensa entre aquello que está más próximo.

Para terminar, es significativo mencionar que en caso de utilizar datos de la WoS los resultados serán distintos. Para obtener resultados óptimos lo ideal sería emplear datos de ambas bases de datos, la de la WoS y la de Scopus, para después cruzarlos y evitar duplicidades. Sin embargo, Scopus ofrece una visión suficientemente completa para observar tendencias a nivel global.

\section{Reconocimientos}

Este artículo se presenta como parte de las actividades obligatorias en el programa de Doctorado en Geomática en la Universidad Politécnica de Valencia con el fin de publicitar los avances en la tesis doctoral, en este caso, dirigida por el profesor Jesús Manuel Palomar Vázquez del Departamento de Ingeniería Cartográfica, Geodésica y Fotogrametría de la misma universidad.

\section{References}

BEARMAN, N., MUNDAY, P., and MCAVOY, D., 2015. Teaching GIS outside of geography: a case study in the School of International Development, University of East Anglia. Journal of Geography in Higher Education, 39(2). DOI: 10.1080/03098265.2015.1010146. 
BOONSTRA, O.W.A., 2009. No place in history geo-ICT and historical science. In Scholten, H.J., van de Velde, R. \& van Manen, N. Geospatial technology and the role of location in science, pp. 87-101, Dordrecht, NL: Springer.

ELLUL, C., 2015. Geography and Geographical Information Science: interdisciplinary integrators. Journal of Geography in Higher Education, 39(2), pp. 191-194. DOI: 10.1080/03098265.2015.1039797.

ELSEVIER, n.d.. Content Policy and Selection. Available: https://www.elsevier.com/solutions/scopus/content/contentpolicy-and-selection [5/1, 2017].

EUROPEAN COMMISSION, 2011. Modernisation of higher education in Europe: funding and the social dimension. Education, Audiovisual and Culture Executive Agency Eurydice. ISBN: 978-92-9201-205-2.

EUROPEAN COMMISSION, 2014. Horizon 2020 in brief. The EU Framework Programme for Research \& Innovation. Publications Office of the European Union, Luxembourg. ISBN: 978-92-79-33057-5. Available: http://ec.europa.eu/programmes/horizon2020/en/h2020-section/europe-changing-world-inclusive-innovative-andreflective-societies [2/20, 2015].

FERRANDINO, J., 2015. Using GIS to apply learning across the undergraduate criminal justice curriculum. Journal of Criminal Justice Education, 26(1), pp. 74-93. DOI: 10.1080/10511253.2014.925567.

GEWIN, V., 2004. Mapping Opportunities. Nature, 427, pp. 376-377. DOI: 10.1038/nj6972-376a.

GOODCHILD, M.F., and JANELLE, D.G., 2010. Toward critical spatial thinking in the social sciences and humanities. GeoJournal, 75, pp. 3-13. DOI: 10.1007/s10708-010-9340-3

JESSOP, M., 2008. The Inhibition of Geographical Information in Digital Humanities Scholarship. Literacy and linguistic computing 23, pp. 39-50. DOI: 10.1093/llc/fqm041

OREGON STATE BOARD OF EDUCATION, 2004. GIS Decision Support Pilot Project. Technical Summary. Available: http://www.oregon.gov/ccwd/word/stateboard.doc [12/14, 2014].

PÉREZ-GÓMEZ, R., 2012. Analyzing and mapping key issues in higher education systems with geo-information related technologies. 4th International Conference on Cartography and GIS, Vol. 1. Albena, June 18-22. Available: http://www.cartography-gis.com/docsbca/Book_Proceedings\%201_2012.pdf [11/12, 2014].

RUMBLEY, L.E., STANFIELD, D.A. and DE GAYARDON, A., 2014. From inventory to insight: making sense of the global landscape of higher education research, training, and publication. Studies in Higher Education, 39(8), pp. 1293-1305. DOI: 10.1080/03075079.2014.949546.

SINGLETON, A.D., WILSON, A.G., and O'BRIEN, O., 2012. Geodemographics and spatial interaction: An integrated model for higher education. Journal of Geographical Systems, 14, pp. 223-241. DOI: 10.1007/s10109-010-0141-5.

SMITH, G., and SMITH, T., 2014. Targeting educational disadvantage by area: continuity and change in urban areas in England 1968-2014. Oxford Review of Education, 40(6), pp. 715-738. DOI: 10.1080/03054985.2014.981436.

SUI, D., 2014. Opportunities and impediments for Open GIS. Transactions in GIS, 18(1), pp. 1-24. DOI: $10.1111 /$ tgis. 12075.

TOBLER, W., 1970. A computer movie simulating urban growth in the Detroit region. Economic Geography, 46(2), pp. 234-240. DOI: 10.2307/143141.

TURI, K.N., CHRISTOPH, M.J., and GRIGSBY-TOUSSAINT, D.S., 2013. Spatial distribution of underweight, overweight and obesity among women and children: Results from the 2011 Uganda demographic and health survey. International Journal of Environmental Research and Public Health, 10(10), pp. 4967-4981. DOI: 10.3390/ijerph10104967.

UNITED NATIONS COMMITTEE OF EXPERTS ON GLOBAL GEOSPATIAL INFORMATION MANAGEMENT, 2014. About UN-GGIM. Available: http://ggim.un.org/about.html [3/1, 2015].

U.S. DEPARTMENT OF LABOR, 2010. Employment and Training Administration - High growth industry profile Geospatial Technology. Available: http://www.doleta.gov/BRG/Indprof/geospatial_profile.cfm [10/14, 2015].

WANG, A., GUO, W., XU, G., JIA, J., and WEN, D., 2009. GIS-Based Educational Decision-Making System. 2009 IEEE International Conference on Grey Systems and Intelligent Services, GSIS 2009, pp. 1198-1202.

XIE, Y., and REIDER, D., 2014. Integration of Innovative Technologies for Enhancing Students' Motivation for Science Learning and Career. Journal of Science Education and Technology, 23, pp. 370-380. DOI: 10.1007/s10956-0139469-1. 